\title{
Identificación del talento musical en escolares de la Comunidad Autónoma de la Región de Murcia en España
}

\author{
Identification of Musical Talent in Schoolchildren of the Autonomous Community of the Region of \\ Murcia, Spain
}

\section{Identificação do talento musical junto a estudantes da Comunidade Autônoma da Região de Murcia na Espanha}

Francisco Rafael Lázaro-Tortosa
Universidad de Murcia
Departamento Métodos de Investigación y Diagnóstico en Educación
Murcia, España
franciscorafael.lazaro@um.es
https://orcid.org/0000-0002-9802-4320
María Marco-Arenas
Universidad de Murcia
Departamento Métodos de Investigación y Diagnóstico en Educación
Murcia, España
maria.marco1@um.es
(iD) https://orcid.org/0000-0003-2988-8827
María Cristina Sánchez-López
Universidad de Murcia
Departamento Métodos de Investigación y Diagnóstico en Educación
Murcia, España
crisalo@um.es

Recibido • Received • Recebido: 12 / 09 / 2019

Corregido • Revised • Revisado: 23 / 06 / 2021

Aceptado • Accepted • Aprovado: 12 / 08 / 2021

\begin{abstract}
Resumen:Este trabajo surge de la creencia en la necesidad de identificar los talentos musicales deescolares, se sigue en este el axioma de la teoría de las inteligencias múltiples de que un verdadero desarrollo integral se consigue atendiendo a todas las inteligencias. La identificación se lleva a cabo en colegios de educación primaria de la Comunidad Autónoma de la región de Murcia, España, seleccionados según un proceso estratificado y polietápico. Las personas participantes son 932 estudiantes con edades comprendidas entre los diez y doce años, de diferentes centros educativos tanto públicos como concertados-privados. El instrumento utilizado ha sido el Test de aptitudes musicales de Seashore (Seashore et al. (2008), a cuyas puntuaciones obtenidas se aplica el modelo de identificación de altas capacidades cognitivas de Castelló y Batlle. Los resultados indican que en esta comunidad autónoma existen estudiantes con recursos intelectuales musicales propios de la alta habilidad, y el tono, el ritmo y el tiempo son las cualidades mejor puntuadas en el talento simple, y el ritmo y el tiempo en el talento complejo.
\end{abstract}

Palabras claves: Talento musical; aptitudes musicales; inteligencias múltiples; educación primaria. 
http://doi.org/10.15359/ree.25-3.17

http://www.una.ac.cr/educare

educare@una.ac.cr

\begin{abstract}
This work arises from the belief in the need to identify the musical talents of schoolchildren; the work follows the axiom of the theory of multiple intelligences that true integral development is achieved attending all the intelligences. The identification was carried out in primary schools of the Autonomous Community of the Region of Murcia (Spain); the schools were selected according to a stratified and multi-stage process. The participants were 932 students between the ages of ten and twelve from different public and private-subsidized educational centers. The instrument used was the Musical Aptitude Test (Seashore et al., 2008), whose scores obtained were analyzed with the Castelló and Batlle's High Cognitive Capability Identification Model. In this autonomous community, the results indicate that there are students with musical intellectual resources of high ability and that the highest-rated qualities are tone, rhythm, and time in simple talent and rhythm and time in complex talent.
\end{abstract}

Keywords: Musical talent; musical ability; multiple intelligence; primary education.

Resumo: Este trabalho surge da crença na necessidade de identificar os talentos musicais das crianças em idade escolar, seguindo o axioma da Teoria das Inteligências Múltiplas, de que um verdadeiro desenvolvimento integral é alcançado atendendo a todas as inteligências. A identificação é realizada nas escolas de Ensino Fundamental da Comunidade Autônoma de la Region de Murcia (Espanha), selecionadas de acordo com um processo estratificado e de várias etapas. Participam 932 estudantes com idades entre dez e doze anos, de diferentes centros educacionais, públicos e privados subsidiados. O instrumento utilizado foi o Teste de Aptidão Musical Seashore et al. (2008), ao qual são aplicadas as pontuações obtidas no Modelo de Identificação de Altas Capacidades Cognitivas de Castelló e Batlle. Os resultados indicam que na Comunidade Autônoma há estudantes com recursos intelectuais musicais típicos de alta habilidade, com tom, ritmo e tempo sendo as qualidades mais bem avaliadas em talentos simples, e ritmo e tempo em talentos complexos.

Palavras-chave: Talento musical; aptidões musicais; inteligências múltiplas; ensino fundamental.

\title{
Introducción
}

La idea inicial de esta investigación surgió de las reflexiones como docentes de educación primaria sobre la calidad en el desarrollo integral de escolares en el aula y en torno a la diferente jerarquía que los sistemas educativos confieren a las áreas de conocimiento. En dicha jerarquía, las que tienen que ver con la expresión y producción artística ocupan el último lugar. En opinión de Robinson (2012) estamos viviendo una revolución global que demanda a todas las organizaciones, y en especial a los sistemas educativos, redirigir la forma de entender y educar nuestras capacidades para darles mejor uso. En su discurso confiere a todo lo artístico un gran valor y propone que la creatividad se convierta en el centro de la formación. Por su parte, el neurocientífico Mora (2017) ahonda en esta propuesta, informando categóricamente que ha llegado el momento de que la educación supere la visión científica del mundo, que asuma lo humanístico como parte fundamental de la enseñanza. 
http://doi.org/10.15359/ree.25-3.17

http://www.una.ac.cr/educare educare@una.ac.cr

"[El conjunto] de las diferentes capacidades y/o aptitudes se relaciona con el concepto de educación integral, según la cual se deben desarrollar todas las potencialidades de una persona para formar individuos cultos, inteligentes, libres y con herramientas para entender el mundo" (Lázaro Tortosa, 2015, p. 1). En la práctica, los actuales planteamientos educativos marginan las aptitudes de las personas por lo que se necesita una visión diferente de la inteligencia y la creatividad humana (Robinson, 2012).

Las ideas del citado filósofo inglés Sir Ken Robinson se ajustan a las propuestas por Gardner (1995) en su teoría de las inteligencias múltiples que revolucionó el mundo psicopedagógico. El punto de partida es considerar que el intelecto está formado por un conjunto de capacidades, aptitudes, habilidades o inteligencias, distintas, semi independientes y todas igual de importantes. Además, cada individuo las desarrolla conforme a su evolución personal, intereses y motivaciones.

Si bien esta teoría ha tenido mucha aceptación desde su formulación, en los últimos años se ha cuestionado debido a la falta de evidencias científicas que la avalen. En este sentido la nueva corriente denominada neuroeducación va más allá de potenciar las diferentes facetas intelectivas humanas. Pretende que la formación se fundamente en el conocimiento del funcionamiento del cerebro, hoy en día tan en auge gracias a los avances tecnológicos de medición de la actividad cerebral. Esta nueva corriente educativa fusiona los conocimientos de la neurociencia, psicología y educación, para perfeccionar el proceso de enseñanza y aprendizaje. Esta dinámica de aprendizaje se basa en proponer todo aquello que se sabe acerca de cómo el cerebro aprende y qué mecanismos lo estimulan en el ámbito educativo (Bueno y Forés, 2018). En esta línea, se promueve, desde una perspectiva biológica, un proceso de enseñanza-aprendizaje integrado que rompe con la visión tradicional (Guillén, 2017), basado en el conocimiento de la maduración de las áreas cerebrales. Ahora sabemos que el éxito de una persona en el desarrollo de su profesión y de su vida depende del equilibrio entre dos tipos de habilidades: las habilidades blandas y las habilidades duras. Las primeras referidas a la parte interpersonal como la flexibilidad, la adaptación, la aceptación... en el aprendizaje. Y las segundas, las duras, se obtienen a través de la educación formal, tradicionalmente en entornos académicos o profesionales y están relacionadas con contenidos propios de la educación formal (Buxarrais Estrada, 2013).

En nuestra reflexión como docentes preocupados por la mejor educación observamos que los conocimientos psicopedagógicos y neurológicos que suponen avances no llegan al aula, que el sistema educativo tiene otros parámetros de organización. Un claro ejemplo queda representado en la última legislación educativa española: la Ley Orgánica para la Mejora de la Calidad Educativa (LOMCE), del año 2013 (Jefatura del Estado, 2013). Esta normativa supone un retroceso en la formación que llamamos humanística. Fomenta las materias relacionadas con las aptitudes lógico-matemáticas y lingüísticas, no promueve y deja reducidas en la carga formativa 
http://doi.org/10.15359/ree.25-3.17

http://www.una.ac.cr/educare

educare@una.ac.cr

escolar las relacionadas con la educación artística y de conocimiento personal y relacional. Una manera de constatar esta realidad es observando el número de horas que la administración legisla para cada una de las materias formativas del currículo. Por ejemplo, en la Comunidad Autónoma, la formación artística contemplada en la Ley vigente para la etapa de primaria supone el $8 \%$ del tiempo escolar semanal en los tres primeros cursos del primer tramo. En los tres cursos restantes del segundo tramo, queda reducida al $4 \%$ del horario semanal (Comunidad Autónoma de la Región de Murcia [CARM], 2014). Esta presencia anecdótica de la formación artística en la etapa de primaria nos lleva a concluir que la tan argumentada educación integral por las personas pensadoras educativas no queda garantizada en las decisiones político educativas. Parece, pues, que es imprescindible que el profesorado observe y potencie las capacidades de sus estudiantes para poner a su alcance retos que vayan mucho más allá de los contenidos y competencias que engloba el currículo formal (Acosta Inchaustegui y Alsina, 2017; Castro et al., 2015). Identificar las capacidades musicales de los grupos escolares puede ser nuestro granito de arena para seguir ahondando y optando por una educación integral, humanista y creativa.

\section{Concepto de talento}

El talento, según Gardner (1995), es un rasgo imprescindible de la inteligencia como potencial biopsicológico o psicobiológico. La característica principal del talento es su especificidad, señal del potencial de la neurología del comportamiento que se expresa en cualquier particularidad existente en una cultura (Genovard Rosselló y Castelló Tarrida, 1990).

Dentro de la denominación de talento se encuentran los talentos especializados, que muestran registros extraordinarios de creatividad y maestría. La genialidad es específica de contextos singulares. Cuando un niño o niña destaca en una o en cualquier combinación de las ocho inteligencias que define Gardner, no quiere decir que destacará en otras. Otro término que engloba al talento es la prodigiosidad, que se define como una forma extrema de talento en un solo ámbito, como por ejemplo, Mozart en el campo musical (Sánchez López, 2006).

\section{Tipos de talentos}

Castelló (1986) analizó los términos de superdotación y de talento. Las personas superdotadas son excepcionales intelectuales, en el sentido de que disponen de un conjunto de características que garantizan un alto nivel de funcionamiento de toda la estructura del intelecto.

Los talentos se caracterizan por rendimientos parciales extraordinarios en algún factor de la mencionada clasificación o en otras áreas no estrictamente intelectuales. El grupo de talentos dependerá directamente de los valores sociales predominantes en un momento dado, ya que pueden existir sujetos con habilidades excepcionales que no gocen de suficiente utilidad o interés socio-cultural, por lo que pasan desapercibidos. (Castelló, 1986, p. 101) 
http://doi.org/10.15359/ree.25-3.17

Por todo ello, Castelló Tarrida y de Batlle Estapé (1998) proponen un protocolo de identificación que se extrae de dos instrumentos de medida ya elaborados y baremados: la Batería de aptitudes diferenciales y generales (BADyG) (Yuste et al., 1998) y el Test de pensamiento creativo de Torrance (Torrance, 1974).

“Hasta la fecha, apenas se dispone de instrumentos de rendimiento máximo (Cronbach, 1949) para la evaluación de las [inteligencias múltiples, excepto] ... la batería de Visser et al. (2006) [que mide la aptitud], ... y las Spectrum Activities o SA de Gardner (1998), ejemplo de 'test de logro o de competencia'" (Cejudo et al., 2017) que se han utilizado en ocasiones para medir diferentes habilidades.

En la actualidad, Pérez et al. (2003) crearon el Inventario de autoeficacia para inteligencias múltiples o IAMI, el único autoinforme de IIMM es desarrollado en un contexto hispanohablante y con una clara vocación de aplicación educativa. Este instrumento mide la confianza de adolescentes para realizar exitosamente actividades relacionadas con las ocho inteligencias múltiples (Pérez y Medrano, 2007).

\section{Clasificación de talentos}

El modelo de Castelló Tarrida y de Batlle Estapé (1998) aporta la siguiente clasificación para diferenciar alumnado superdotado y talentoso según la siguiente tipología:

El talento simple lo tiene aquel alumnado que alcanza un percentil de 95 o superior en una sola habilidad intelectual (por ejemplo, verbal o matemática). La combinación de dos o más talentos simples se define como talento múltiple.

El talento complejo lo tiene aquel alumnado que obtiene puntuaciones de 80 o superiores en diferentes habilidades intelectuales. Dentro de esta categoría se puede diferenciar el talento académico que resulta de la combinación de razonamiento verbal, razonamiento lógico y memoria. El talento figurativo que resulta de la combinación de razonamiento lógico y espacial; y por último, el talento figurativo-artístico que resulta de la combinación del talento figurativo e incluye la creatividad. (Castelló Tarrida y de Batlle Estapé, 1998).

El talento conglomerado resulta de la combinación de un talento complejo con cualquier otro tipo de talento, es la combinación mínima que resulta de un talento complejo más uno simple.

El talento superdotado es aquel alumnado que consigue como mínimo un percentil de 70 o 75 en todas las habilidades evaluadas.

\section{Talentos musicales}

Gardner (1995) define el talento musical como una habilidad extraordinaria para apreciar sonidos, ritmos y timbres, discriminarlos, transformarlos y expresarse con ellos. Dicho talento suele ser difícil de identificar, puesto que no todas las personas tienen el mismo grado de 
http://doi.org/10.15359/ree.25-3.17

http://www.una.ac.cr/educare

educare@una.ac.cr

habilidad musical en las mismas dimensiones, sino que destacan en unas más que en otras. En Sánchez López (2006) se localizan las indicaciones que pueden identificar los talentos musicales: (a) escucha y responde con gran interés a las variedades musicales; (b) disfruta y busca momentos para escuchar música o sonidos que le rodean; (c) reconoce y debate sobre diferentes estilos musicales; (d) colecciona grabaciones y bibliografía sobre la música; (e) suele tocar instrumentos musicales; $(\mathrm{f})$ se divierte con el uso del vocabulario y notaciones musicales; (g) desarrolla con facilidad una estructura personal para escuchar música; (h) se divierte improvisando y tocando sonidos; (i) muestra interés en carreras relacionadas con la música; (j) manifiesta potencial para crear composiciones musicales.

Identificar el talento musical en los primeros años de escolaridad puede proporcionarnos una información de gran interés para tomar decisiones sobre cómo plantear programas de formación de las capacidades musicales. Martín López (2006) propone que ello se debe hacer de manera conjunta entre el personal docente y la familia. Su investigación se centró en la identificación del talento musical y su evolución desde la etapa de infantil hasta el último curso de primaria. Los resultados mostraron que el porcentaje de alumnado talentoso en infantil es elevado y que va disminuyendo a medida que se avanza en edad, pues se observa que en el último año cero estudiantes mostraban los índices de talento musical. La explicación sencilla a estos resultados enlaza con nuestra creencia de identificar las aptitudes musicales en escolares es de interés para fomentar su desarrollo en el conjunto de una educación integral. Identificar el talento musical se debería hacer desde la etapa de infantil como afirman Castelló Tarrida y de BatlleEstapé (1998), Gardner (1983), Gardner et al. (2000) y Renzulli (1977).

En el ámbito de la etapa de Educación Primaria interesa citar la investigación (Lázaro Tortosa, 2015) sobre identificación de talentos musicales que establece la relación entre aptitudes musicales e intelectuales y rasgos de personalidad.

\section{Proyecto Spectrum}

En relación con las capacidades intelectuales, las inteligencias múltiples de Gardner y el talento musical, es obligado presentar brevemente el Proyecto Spectrum (Gardner et al., 2000), modelo educativo basado en la teoría de Piaget, que entendía la inteligencia como un proceso de construcción de conjuntos de estructuras cognitivas cada vez más fuertes. Este proyecto, creado en el año 1984, se dedica al desarrollo de un enfoque alternativo del currículo académico y de su evaluación, destacando la importancia de reconocer, promover y fomentar las diferentes capacidades cognitivas que el alumnado posee (Valera Calvo y Plasencia Cruz, 2006).

En el Proyecto Spectrum encontramos la relación de capacidades claves que, según Campell (2000), posee un talento musical (véase Tabla 1). 
http://doi.org/10.15359/ree.25-3.17

Tabla 1: Capacidades claves del talento musical según el Proyecto Spectrum

\begin{tabular}{lll}
\multicolumn{1}{c}{ Percepción } & \multicolumn{1}{c}{ Producción } & \multicolumn{1}{c}{ Composición } \\
\hline - Sensible a la dinámica. & - Mantiene el tono preciso. & - Realiza composiciones sencillas. \\
- Sensible al compás y ritmo. & - Es expresivo al cantar o tocar & - Crea un sistema sencillo de notación. \\
- Discrimina el tono. & instrumento. & \\
- Identifica estilos musicales. & - Mantiene el compás y ritmo. & \\
- Reconoce instrumentos y sus & - Recuerda y produce las & \\
sonidos. & propiedades musicales. & \\
\hline
\end{tabular}

Nota: Elaboración propia a partir de Campell (2000, p.145).

Por último, nos parece de gran interés a portar las características principales de las personas con talento musical que encontramos detalladas en Lancaster (2003) y quedan enumeradas en la Tabla 2. Son una guía extraordinaria para aquel personal docente motivado en la detección de estudiantes que sobresalen por sus aptitudes musicales.

Tabla 2: Características de los talentos musicales respecto a las aptitudes musicales

\begin{tabular}{ll}
\hline Aptitudes musicales & \multicolumn{1}{c}{ Características } \\
\hline \multirow{3}{*}{ Tono } & - Diferencian los agudos y los graves. \\
& - Entienden la relación entre los tonos. \\
& - Identifican intervalos cuando dos tonos suenan de forma simultánea o correlativa. \\
& - Reconocen las diferencias entre los intervalos. \\
& - Pueden identificar sonidos aislados, sin referencia. \\
\hline \multirow{3}{*}{ Ritmo } & - Repiten con facilidad patrones rítmicos escuchados. \\
& - Reconocen un patrón rítmico sin el uso de notación musical. \\
& - Identifican estructuras de patrones rítmicos. \\
& - Poseen un gran razonamiento espacial. \\
\hline \multirow{3}{*}{ Melodía y armonía } & - Memorizan y recuerdan melodías y armonías cada vez más complejas. \\
& - Identifican los cambios que se producen al poner una melodía nueva sobre una \\
& armonía previamente escuchada. \\
\hline \multirow{3}{*}{ Timbre } & - Distinguen fácilmente las características tímbricas de los instrumentos. \\
& - Memorizan sucesiones de timbres. \\
& - Perciben las sutiles diferencias tímbricas entre instrumentos similares. \\
\hline \multirow{2}{*}{ Intensidad } & - Reconocen las amplitudes de los sonidos. \\
& - Reproducen diferentes grados de intensidad.
\end{tabular}

Nota: Elaboración propia a partir de Lancaster (2003). 
http://doi.org/10.15359/ree.25-3.17

http://www.una.ac.cr/educare

educare@una.ac.cr

\section{Método}

\section{Participantes}

Las personas participantes de esta investigación son escolares de edades comprendidas entre los diez y doce años de la Comunidad Autónoma de la Región de Murcia (España). La muestra se extrajo siguiendo un proceso estratificado y polietápico, en el que las unidades de primera etapa fueron las comarcas naturales de la Comunidad Autónoma de la Región de Murcia (España); las de segunda, los municipios; las de tercera, los centros escolares; y las de cuarta y última etapa, las aulas de educación primaria.

Para la selección de las unidades en los niveles de primaria, se utilizó un procedimiento con probabilidad proporcional, lo que da lugar a una muestra autoponderada que simplificó los análisis posteriores. Para un nivel de confianza del 95,5\%, el proceso de muestreo seguido nos proporcionó una muestra estadísticamente representativa con un margen de error del $\pm 3,2 \%$.

La muestra definitiva quedó constituida por 932 estudiantes (488 hombres y 444 mujeres) con edades comprendidas entre los diez y los doce años, pertenecientes a catorce centros de educación infantil y primaria (nueve públicos y cinco concertados) de la Comunidad Autónoma.

\section{Instrumento}

Para la evaluación de las aptitudes musicales se utilizó el Test de aptitudes musicales de Seashore (Seashore et al., 2008), tradicionalmente utilizado en España. Los resultados obtenidos se expresaron en puntuaciones directas y percentiles. El test ofrece medidas separadas sobre seis dimensiones musicales: tono, intensidad, ritmo, sentido del tiempo, timbre y memoria tonal. La elección de esta prueba surgió al revisar diferentes bases de datos, pues resultó ser la más implementada en el ámbito educativo tanto en España como en Norteamérica (Asprilla, 2015).

Así pues, en la identificación del talento musical se han utilizado los resultados del test de Seashore aplicando el modelo de identificación de Castelló Tarrida y de Batlle Estapé (1998) y se encontraron estudiantes con recursos intelectuales musicales propios del superdotado y talento.

Para la identificación de la alta habilidad se han considerado todas las aptitudes musicales, se ha realizado la identificación de forma individual en cada una de ellas. Los indicadores que han dirigido la identificación son:

Talento simple musical: estudiantes que alcanzan un percentil igual o superior a 95 en la media aritmética de las seis aptitudes musicales evaluadas.

Talento complejo musical: estudiantes que alcanzan un percentil igual o superior a 80 en la media aritmética de las seis aptitudes musicales. 
http://doi.org/10.15359/ree.25-3.17

Superdotado musical: estudiante que alcanza un percentil igual o superior a 70 en el resultado de la media aritmética de las seis aptitudes musicales valoradas.

\section{Resultados}

\section{Talentos musicales}

La frecuencia de estudiantes que cumplen dichos indicadores (percentil igual o superior a 95 en las seis aptitudes musicales evaluadas) es la siguiente:

- Talento simple: 0

- Talento complejo: 10

- Superdotado: 29

Así pues, la frecuencia más alta en los participantes corresponde al nivel superdotado musical, seguida del talento complejo musical. Ningún participante ha obtenido en todas las variables musicales evaluadas un percentil igual o superior a 95, es de ahí que la frecuencia en el talento musical simple sea 0.

\section{Talentos simples musicales}

En la Tabla 3 se ofrecen las frecuencias del estudiantado que ha obtenido en la prueba musical un percentil igual o superior a 95, así como inferior a 95 en cada uno de los aspectos musicales valorados.

Tabla 3: Distribución de estudiantes con recursos intelectuales de talento simple musical

\begin{tabular}{rrrrrrr}
\hline & Tono & Intensidad & Ritmo & Tiempo & Timbre & Memoria \\
\hline$\geq 95$ & 78 & 6 & 44 & 40 & 11 & 21 \\
$<95$ & 854 & 926 & 888 & 892 & 921 & 911 \\
\hline
\end{tabular}

Nota: Elaboración propia.

Como se desprende de los resultados que se reflejan en la Tabla 3, la aptitud musical que ha logrado una mayor puntuación en el talento simple musical (puntuación igual o superior a $95)$ es el tono (8,3\%), seguido por el ritmo (4,9\%) y tiempo (4,2\%). Las aptitudes que obtienen menores puntuaciones son intensidad $(0,6 \%)$ y timbre $(1,1 \%)$. 
http://doi.org/10.15359/ree.25-3.17

http://www.una.ac.cr/educare

educare@una.ac.cr

\section{Talentos complejos musicales}

En la Tabla 4 se ofrecen las frecuencias del alumnado que ha obtenido en la prueba musical un percentil igual o superior a 80 e inferior a 80 en cada uno de los aspectos evaluados.

Tabla 4: Distribución de estudiantes con recursos intelectuales de talento complejo musical

\begin{tabular}{rcccccc}
\hline & Tono & Intensidad & Ritmo & Tiempo & Timbre & Memoria \\
\hline$\geq 80$ & 142 & 41 & 202 & 173 & 49 & 91 \\
$<80$ & 780 & 891 & 730 & 759 & 883 & 841 \\
\hline
\end{tabular}

Nota: Elaboración propia.

Las dimensiones que han logrado mayores puntuaciones en el talento complejo musical (puntuación igual o superior a 80 ) son ritmo con $22 \%$ y tiempo con un $18 \%$. Las aptitudes musicales de intensidad $(0,4 \%)$ y timbre $(0,5 \%)$ son las que menores puntuaciones alcanzan.

\section{Superdotados musicales}

En la Tabla 5 se ofrecen las frecuencias del alumnado que ha obtenido en la prueba musical un percentil igual o superior a 70 e inferior a 70 en cada uno de los aspectos valorados.

Tabla 5: Distribución de estudiantes con recursos intelectuales superdotados musicales

\begin{tabular}{ccccccc}
\hline & Tono & Intensidad & Ritmo & Tiempo & Timbre & Memoria \\
\hline$\geq 70$ & 219 & 113 & 303 & 251 & 116 & 167 \\
$<70$ & 713 & 819 & 629 & 681 & 816 & 765 \\
\hline
\end{tabular}

Nota: Elaboración propia.

Tal y como se refleja en la Tabla 5, la aptitud musical que ha logrado una mayor puntuación en el superdotado musical (puntuación igual o superior a 70 ) es el tono (8,3\%), seguido por el ritmo (4,9\%) y tiempo (4,2\%). Las variables que obtienen menores puntuaciones son intensidad $(0,6 \%)$ y timbre $(1,1 \%)$.

\section{Resumen talentos musicales}

A modo de resumen, en la Tabla 6 se muestra la frecuencia de estudiantes identificados de modo general y considerando la especificidad de las aptitudes musicales evaluadas (talentos simples, complejos y superdotados). 
http://doi.org/10.15359/ree.25-3.17

http://www.una.ac.cr/educare educare@una.ac.cr

Tabla 6: Resumen talentos musicales

\begin{tabular}{cccccccc}
\hline Talentos & Musical & Tono & Intensidad & Ritmo & Tiempo & Timbre & Memoria \\
\hline Simple & 0 & 78 & 6 & 44 & 40 & 11 & 21 \\
Complejo & 10 & 142 & 41 & 202 & 73 & 49 & 91 \\
Superdotado & 29 & 219 & 113 & 303 & 251 & 116 & 167 \\
\hline
\end{tabular}

Nota: Elaboración propia.

\section{Conclusiones e implicaciones educativas}

En la Comunidad Autónoma de la región de Murcia hay escolares con recusos intelectuales comunes a la alta habilidad, estos corresponden al 0,1\% de la población total y el 4,1\% de estudiantes de esta investigación. Del grupo evaluado de escolares, 39 participantes poseen un gran potencial en las habilidades musicales valoradas, 10 poseen recursos intelectuales musicales relacionados del talento complejo musical y 29 del perfil se superdotación.

Estos datos relativos a la inteligencia son muy significativos a la hora de compararlos con los diferentes personas expertas que han valorado las altas capacidades relacionadas con la música. Las personas estudiosas sobre este tema apuntan que el número de escolares con superdotación es el 2,3\%, el 0,05\% se corresponden con talentos complejos y el 0,000003\% para talentos simples (Martín López, 2006).

En esta investigación los resultados indican que el 3\% del alumnado es superdotado musical, esto es un $30 \%$ más de lo que indican las personas expertas sobre los cognitivos. El estudiantado que posee un talento simple musical asciende hasta el 1,1\%, este es un $21,00 \%$ más respecto al intelectual, es decir, aumentan más de 21 veces los talentos simples musicales que los intelectuales. Por todo ello, estos resultados manifiestan que en esta región hay más porcentaje de talentos musicales que intelectuales (Lázaro Tortosa, 2015).

Hernández Bravo et al. (2007) consideran vital diagnosticar tempranamente el talento musical del alumnado para prestarles una respuesta educativa lo más adecuada a sus necesidades. El alumnado con talento musical posee un factor general de habilidad musical, presta mayor atención de forma específica a los estímulos auditivos abstractos $y$, además, es muy creativo.

La educación artístico-musical debería convertirse en un eje fundamental del sistema educativo. Esta materia desarrolla un rol imprescindible en el proceso de la formación integral de las personas. Además, desplegar en la población escolar las aptitudes musicales es darles la oportunidad de potenciar la actividad cognitiva general del cerebro. Por todo ello, el personal docente especialista en educación musical de los colegios tiene que fomentar esta disciplina, aun sabiendo que cada vez tiene menos carga lectiva en el sistema educativo español. 
http://doi.org/10.15359/ree.25-3.17

http://www.una.ac.cr/educare

educare@una.ac.cr

El neurocientífico Dr. Zatorre y sus colaboradores nos vienen informando desde finales del siglo pasado de las potencialidades del cerebro en relación con la localización de las zonas de procesamiento musical: cuando se está procesando música se activa toda una red de centros neuronales distribuidos por ambos hemisferios (Blood y Zatorre, 2001). De forma muy didáctica en sus conferencias explican que el resultado de dicha activación es como si se encendieran múltiples semáforos por toda la superficie del cerebro. "La actividad musical implica casi todas las regiones del cerebro sobre las que tenemos conocimientos, y casi todo el subsistema neurológico" (Levitin, 2018, p. 94). Por lo tanto, la actividad musical tiene enormes beneficios para hacer más potentes las conexiones neuronales que dan como resultado mayor inteligencia en su conjunto. También son muy interesantes las investigaciones que concluyen que las estructuras corticales que hasta ahora se identificaban como áreas exclusivas del procesamiento del lenguaje, no lo son tanto ya que en ellas también se procesa información musical (Koelsch et al., 2002).

En esta línea, medir, evaluar y estimular las aptitudes musicales en las etapas de infantil y primaria como propone Gardner (2005) al referirse a la inteligencia musical tiene implicaciones para otras actividades como en el proceso de aprender a leer. Así, interesaría para próximas investigaciones centrarse en estudiar en escolares la relación entre aptitudes musicales y la adquisición del lenguaje.

Si tuviésemos autoridades políticas interesadas en la formación artístico-musical de la población escolar sería muy fácil hacerles ver dos importantes cuestiones que ahora solo se contemplan en el reducido ámbito de la docencia de la música: por un lado, el hecho de que la educación musical es un dominio cognoscitivo que proporciona una forma de conocer única. Por otro lado, que no bastan los centros especiales de formación musical como conservatorios o escuelas de música, que toda la población escolar posee cualidades musicales al igual que otras cualidades intelectuales que deben desarrollarse.

Martín López (2006), en su investigación sobre identificación de talentos musicales en educación infantil y primaria, postula que es necesario intervenir en el desarrollo del talento de forma temprana, y propone que se desarrolle y trabaje para que permanezca a lo largo de la vida. Por ello, se debería crear un protocolo autonómico o estatal similar al de identificación de altas capacidades cognitivas, pero adaptado al talento musical. Como explica Buxarrais Estrada (2013), el éxito de las personas tanto personal como profesional dependerá del equilibrio de las habilidades blandas y duras, ya que favorece el sistema académico al potenciar todas las cualidades del alumnado para el desarrollo integral. En este sentido, los sistemas educativos perpetúan el error de centrarse de forma exclusiva en las capacidades lingüísticas y lógicas (habilidades duras), lo que hace expresar a Gardner que puede suponer una estafa para los individuos que tienen otras capacidades (Gardner, 2005). Consecuencia directa de que los sistemas educativos están obsoletos y anticuados, ya que solo se centran en las capacidades 
http://doi.org/10.15359/ree.25-3.17

lógico matemáticas, dejando de lado tanto las habilidades creativas como las referidas a la parte interpersonal de sus escolares (habilidades blandas) tales como la flexibilidad, la adaptación, la aceptación, etc. (Robinson, 2012).

Por último, el Dr. Mora Teruel nos transmite que la estructura de nuestro cerebro es la misma desde hace quince mil años, que lo que nos cambia es la educación. Y que todo lo que el ser humano hace o siente depende del funcionamiento del cerebro (Mora, 2017). Consideramos, por lo tanto, que atender a la identificación de los talentos musicales es profundizar en el conocimiento del cerebro y tener mejor preparación para afrontar los retos de la formación artístico-musical de nuestra población escolar.

Así pues, ha quedado demostrado tanto por personal científico como por personal pedagogo que la identificación del talento musical, así como su posterior desarrollo, tanto en los centros educativos como fuera de ellos, es vital para el desarrollo integral de escolares. El personal docente, como la familia y el propio contexto que rodea al alumnado, debe garantizar esta formación de sus habilidades duras y blandas por igual, creando así un futuro más favorable para toda la sociedad.

\section{Declaración de Material complementario}

Este artículo tiene disponible, como material complementario:

-La versión preprint del artículo en https://doi.org/10.5281/zenodo.4041970

\section{Referencias}

Acosta Inchaustegui, Y. y Alsina, Á. (2017). Conocimientos del profesorado sobre las altas capacidades y el talento matemático desde una perspectiva inclusiva. Números. Revista de Didáctica de las Matemáticas, 94, 71-92. http://funes.uniandes.edu.co/9348/1/ Acosta2017Conocimientos.pdf

Asprilla, L. I. (2015). Educar en música: Una aproximación crítica al talento y a la educación musical. Aula, 21, 63-83. https://doi.org/10.14201/aula2015216383

Blood, A. J. y Zatorre, R. J. (2001). Intensely pleasurable responses to music correlate with activity in brain regions implicated in reward and emotion. Procedings of the National Academy of Sciences, 98(20), 11818-11823. https://doi.org/10.1073/pnas.191355898

Bueno, D. y Forés, A. (2018). 5 principios de la neuroeducación que la familia debería de saber y poner en práctica. Revista Iberoamericana de Educación, 78(1), 13-25. https://doi. org/10.35362/rie7813255

Buxarrais Estrada, M. R. (2013). Nuevos valores para una sociedad. Un cambio de paradigma en Educación. Edetania: Estudios y propuestas socio-educativas, (43), 53-65. https://dialnet. unirioja.es/ejemplar/342477 
http://doi.org/10.15359/ree.25-3.17

http://www.una.ac.cr/educare

educare@una.ac.cr

Campell, B. (2000). Los muchos rostros del Spectrum. En H. Gardner y D. H. Feldman y M. Krechevsky (Comps.), El Proyecto Spectrum. Tomo 1: Construir sobre las capacidades infantiles (131-159). Morata.

Castelló, A. (1986). Bases para la identificación del superdotado [Tesis de licenciatura]. Universidad Autónoma de Barcelona.

Castelló Tarrida, A. y de Battle Estapé, C. (1998). Aspectos teóricos e instrumentales en la identificación del alumnado superdotado y talentoso, propuesta de un protocolo. Grialibros.

Castro, E., Ruiz-Hidalgo, J. F. y Castro-Rodríguez, E. (2015). Retos, profesores y alumnos con talento matemático. Aula, 21, 85-104. http://dx.doi.org/10.14201/aula20152185104

Cejudo, J., Losada, L. y Pérez-González, J. C. (2017). Inteligencias múltiples y su relación con inteligencias cognitiva y emocional en adolescentes. Universitas Psychologica, 6(3), 1-13. $\quad$ https://revistas.javeriana.edu.co/files-articulos/UPSY/16-3\%20 (2017)/64752604008/\#redalyc 64752604008 ref49

Comunidad Autónoma de la Región de Murcia (CARM). (6 de septiembre de 2014). Decreto 198/2014, de 5 de septiembre, por el que se establece el currículo de la Educación Primaria en la Comunidad Autónoma. Boletín Oficial de la Región de Murcia, Número 206, pp. 33054-33556.

Gardner, H. (1983). Frames of mind. The theory of multiple inteligences. Basic Books.

Gardner, H. (1995). Inteligencias múltiples. La teoría en la práctica. Paidós.

Gardner, H. (2005). Inteligencias múltiples. La teoría en la práctica. Paidós.

Gardner, H., Fieldman, D. H. y Krechevscky, M. (Comps.). (2000). El Proyecto Spectrum. Tomo 1: Construir sobre las capacidades infantiles. Morata.

Genovard Rosselló, C. y Castelló Tarrida, A. (1990). El límite superior. Aspectos psicopedagógicos de la excepcionalidad intelectual. Pirámide.

Guillén, J. C. (2017). Neuroeducación en el aula. De la teoría a la práctica. CreateSpace.

Hernández Bravo, J. R., Hernández Bravo, J. A. y Milán Arellano, M. Á. (2007). La creatividad asociada al talento musical en alumnos superdotados. Respuestas educativas. Ensayos, (22), 83-97. https://dialnet.unirioja.es/servlet/articulo?codigo $=2591548$

Jefatura del Estado. (Martes 10 de diciembre de 2013). Ley Orgánica 8/2013, de 9 de diciembre, para la mejora de la calidad educativa. Boletín Oficial del Estado., num. 295, pp. 97858-97921.

Koelsch, S., Gunter T. C., Cramon, D. Y. V., Zysset, S, Lohmann, G. y Friederici, A. D. (2002). Bach Speaks: A cortical "language-network" serves the processing of music. Neurlmage 17(2), 956-966. https://doi.org/10.1006/nimg.2002.1154 
http://doi.org/10.15359/ree.25-3.17

http://www.una.ac.cr/educare educare@una.ac.cr

Lancaster, H. (2003). Identifying the gifted in music. Thai National Center Bangkok, 1- 12.

Lázaro Tortosa, F. (2015). Relación de aptitudes musicales, intelectuales y rasgos de personalidade identificación del talento musical en escolares de diez a doce años [Tesis doctoral]. Universidad de Murcia.

Levitin, D. J. (2018). Tu cerebro y la música. El estudio científico de una obsesión humana. RBA.

Martín López, E. (2006). Aptitudes musicales y atención en niños entre diez y doce años [Tesis doctoral]. Universidad de Extremadura. http://dehesa.unex.es/handle/10662/501

Mora, F. (2017). Neuroeducación lo que nos enseña el cerebro. Alianza Editorial.

Pérez, E. R. y Medrano, L. (2007). Inventario de autoeficacia para inteligencias múltiples revisado: Un estudio de validez de criterio. Avances en Medición, 5(1), 105-114. https://www.humanas. unal.edu.co/lab psicometria/application/files/6616/0463/3543/Vol 5. Inventario De Autoeficacia Para Inteligencias Mltiples Revisado - Un Estudio De Validez De Criterio.pdf\#: :text=El\%20Inventario\%20de\%20Autoeficacia\%20para,asociadas\%20 con\%20las\%20inteligencias\%20m\%C3\%BAltiples

Pérez, E., Beltramino, C. y Cupani, M. (2003). Inventario de autoeficacia para inteligencias múltiples. Fundamentos teóricos y estudios psicométricos. Evaluar, 3(1), 36-60. https:// revistas.unc.edu.ar/index.php/revaluar/article/view/606/575

Renzulli, J. S. (1977). The enrichment triad model: A guide for developing defensible programs for the gifted and talented. Creative Learning Press.

Robinson, K. (2012). Busca tu elemento. Aprender a ser creativo individual y colectivamente. Empresa Activa.

Sánchez López, M C. (2006). Configuración cognitivo-emocional en alumnos de altas habilidades [Tesis doctoral]. Universidad de Murcia. https://www.tesisenred.net/ handle/10803/10993\#page $=1$

Seashore, C. E., Lewis D. y Seatveit J. G. (2008). Test de aptitudes musicales de Seashore: Manual. TEA.

Torrance, E. P. (1974). The Torrance tests of creative thinking-norms-technical manual research edition-verbal tests, forms $A$ and $B$-figural tests, forms $A$ and $B$. Personnel Press.

Valera Calvo, C. y Plasencia Cruz, I. del C. (2006). El Proyecto Spectrum: Ampliación y actividades de aprendizaje de ciencias en el primer ciclo de educación primaria. Revista de Educación, 339, 947-958. https://redined.mecd.gob.es/xmlui/handle/11162/69123

Yuste, C., Yuste, D., Martínez R., y Gálvez J. L. (1998). Batería de aptitudes diferenciales y generales $B A D y G E_{3}$. CEPE. 